\title{
Spitzer Space Telescope observations of the nucleus of comet 67P/Churyumov-Gerasimenko*
}

\author{
P. L. Lamy ${ }^{1}$, I. Toth ${ }^{1,2}$, O. Groussin ${ }^{1}$, L. Jorda ${ }^{1}$, M. S. Kelley ${ }^{3}$, and J. A. Stansberry ${ }^{4}$ \\ 1 Laboratoire d'Astrophysique de Marseille, UMR6110, CNRS/Université de Provence, Technopôle de Marseille-Etoile, \\ 38 rue Frédéric Joliot-Curie, 13388 Marseille Cedex 13, France \\ e-mail: philippe.lamy@oamp.fr \\ 2 Konkoly Observatory, PO Box 67, 1525, Hungary \\ 3 Department of Physics, University of Central Florida, Orlando, FL 32816, USA \\ 4 Steward Observatory, University of Arizona, Tucson, AZ 85721, USA
}

Received 4 February 2008 / Accepted 18 May 2008

\begin{abstract}
Context. Comet 67P/Churyumov-Gerasimenko is the target of the Rosetta mission, and an early characterization of its nucleus is required to assist in preparing the orbital strategy of the spacecraft, the delivery of the Philae surface module and the science operations. We detected the nucleus using the Hubble Space Telescope in March 2003, but had to assume an albedo to derive its size from its observed magnitudes.

Aims. It is important to derive an additional constraint for independently determining both the comet size and albedo.

Methods. We implemented the well-known "radiometric method", which combines visible and infrared photometry. Sixteen thermal images of 67P/C-G were obtained by the Multiband Imaging Photometer (MIPS) $24 \mu$ m channel of the Spitzer Space Telescope (SST) on 25 February 2004: the observations spanned a time interval of $\sim 12.5 \mathrm{~h}$, which sampled the rotational light curve of its nucleus. The comet was then outbound at a heliocentric distance of $4.48 \mathrm{AU}$, at a distance of $4.04 \mathrm{AU}$ from SST, and at a solar phase angle of $12.1^{\circ}$. The nucleus conspicuously appeared as a bright point source superimposed on a dust trail; it was necessary to apply the point-spread function fitting technique using an adequate model of the trail to correctly determine the thermal flux from the nucleus. The data were analyzed using a standard thermal model that incorporated the thermal inertia.

Results. Our preferred solution with a low thermal inertia has overall dimensions measured along the principal axis of 4.40-5.20 km, $4.16-4.30 \mathrm{~km}$, and 3.40-3.50 km, corresponding to an effective radius of a sphere with the same volume in the range of 1.93-2.03 km. Larger values of thermal inertia produce larger sizes but the effective radius cannot exceed $\sim 2.3 \mathrm{~km}$. The albedo is in the range 0.039-0.043, remarkably consistent with the canonical value of 0.04 for cometary nuclei. The success of the landing of the Philae surface module remains critically dependent upon the bulk density of the nucleus: it would be safe if close to $0.35 \mathrm{~g} \mathrm{~cm}^{-3}$, but a larger value, for instance $0.5 \mathrm{~g} \mathrm{~cm}^{-3}$, would present some risks.
\end{abstract}

Key words. comets: general - comets: individual: 67P/Churyumov-Gerasimenko - techniques: image processing

\section{Introduction}

The Rosetta spacecraft was due to be launched in February 2003. Difficulties with the new Ariane V launcher led to its cancellation and forced ESA to find new potential targets because it would have been no longer possible to rendezvous with 46P/Wirtanen, the original target. Only 67P/ChuryumovGerasimenko emerged as a suitable candidate on the basis of orbital considerations (Schwehm 2003; Ellwood \& Schwehm 2004). However the size of the nucleus was a highly critical parameter for the safe landing of the Philae surface module since it will fall freely onto the surface following its separation from the orbiter. Fortunately, the comet was able to be observed in early March 2003 by the Hubble Space Telescope. We isolated the nucleus of 67P/C-G from its surrounding coma and measured its light curve applying a technique that benefitted from the high spatial resolution of HST. We determined a rotational period of $12.4 \mathrm{~h}$ and, assuming a standard cometary albedo of 0.04 , a mean

\footnotetext{
* Based on observations made with the NASA Spitzer Space Telescope.
}

effective radius of $1.98 \pm 0.02 \mathrm{~km}$ (Lamy et al. 2006), significantly larger than that of 46P/Wirtanen (Lamy et al. 1998a). The landing gear of Philae was reinforced accordingly, and the Rosetta spacecraft was successfully launched on 2 March 2004. It is now travelling to meet with comet $67 \mathrm{P} / \mathrm{C}-\mathrm{G}$ in May 2014 at a heliocentric distance of $4.5 \mathrm{AU}$, some 15 months before the comet's perihelion passage.

Monte Carlo simulations of the landing of Philae indicate that this operation remains a critical issue (Hilchenbach et al. 2004), see Fig. 1. In a broad stroke, the range of altitudes for delivery of the lander is determined by the impact velocity capability of the landing gear (upper limit) and by the separation velocity of the separation mechanism (lower limit), and is inversely proportional to the mass of the nucleus which is dependent on its size and density. The density of cometary nuclei cannot be directly measured but can be inferred from different processes (non-gravitational forces, disruption). The range $0.5-1.2 \mathrm{~g} \mathrm{~cm}^{-3}$ appears to be firmly established and is consistent with our present understanding of the "under-dense" structure of nuclei (Weissman et al. 2004). 


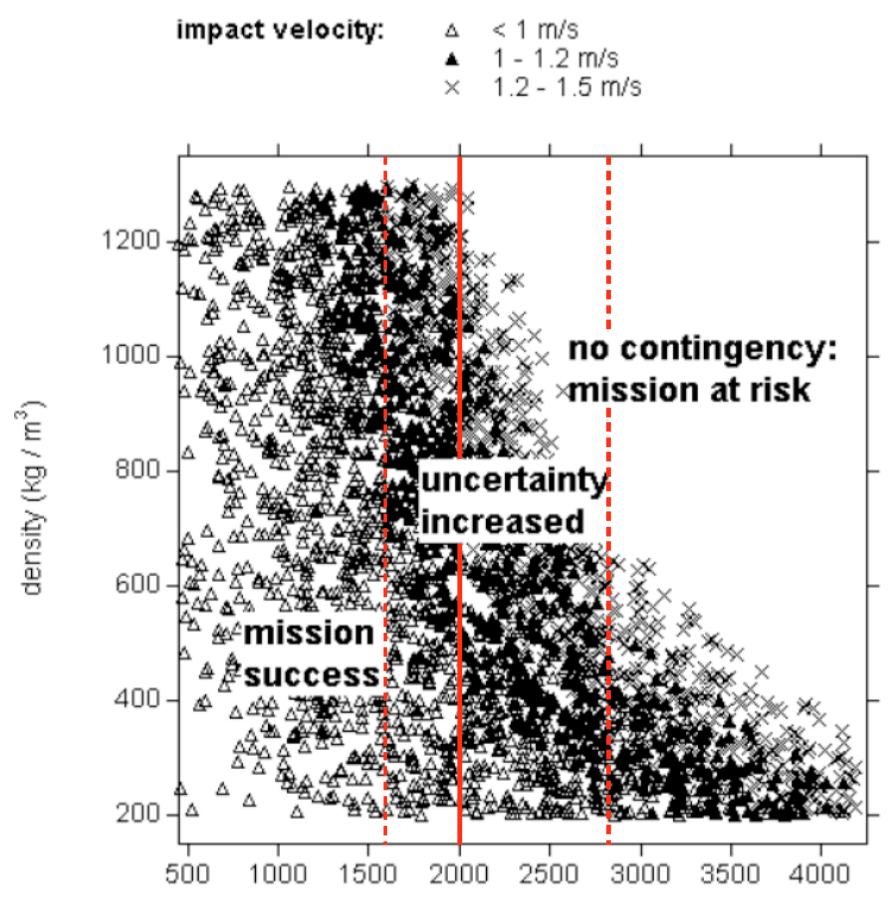

radius (m)
This is because the thermal emissivity is close to 1 . Thanks to Director Discretionary time, we measured the thermal emission of the nucleus of 67P/C-G at $24 \mu \mathrm{m}$ with the Multiband Imaging Photometer (MIPS) instrument of the Spitzer Space Telescope (SST). We presented a preliminary analysis of the data based on the performance of MIPS as calibrated in 2004 (Lamy et al. 2004b, 2007). Our understanding of this instrument improved significantly after the in-depth characterization performed by Engelbracht et al. (2007), and the present detailed analysis of our SST observations benefits from this progress, in particular the proper determination of the point-spread function (PSF).

$67 \mathrm{P} /$ Churyumov-Gerasimenko is a Jupiter-family comet whose orbital elements have undergone significant variations following a series of close encounters with Jupiter (Carusi et al. 1985 ). From $\sim 1600$ to $\sim 1959$, both the perihelion and aphelion distances of the comet (respectively $q$ and $Q$ ) increased, while its inclination $i$ continued to decrease slightly. Then a significant reduction in both $q$ (from $\sim 2.7$ to $\sim 1.3 \mathrm{AU}$ ) and $i$ (from $\sim 28^{\circ}$ to $\sim 7^{\circ}$ ) then occured, followed by a complete reorientation of the nodal line. These events probably triggered extra activity of the comet, leading to its discovery in 1969 . The present orbit is characterized by a period of 6.57 years, $q=1.292 \mathrm{AU}, e=0.632$, and $i=7.12^{\circ}$. The comet has now completed only 7 perihelion passages on its new orbit ( $q \sim 1.3 \mathrm{AU})$.

\section{Observations and preprocessing}

Comet 67P/C-G was successfully observed by the $24 \mu \mathrm{m}$ channel of the Multiband Imaging Photometer (Rieke et al. 2004) of the Spitzer Space Telescope (Werner et al. 2004) in Photometry/Super Resolution mode (native pixel scale $\approx 2.5 \times$ $2.6 \operatorname{arcsec}_{\mathrm{pixel}}^{-1}$ ), offering an approximate field-of-view of $7^{\prime} \times 8^{\prime}$. The observations were acquired on 25 February 2004, when the comet was at $4.48 \mathrm{AU}$ outbound from the Sun. The observing program consisted of 16 visits separated by variable time steps of $0.5,1$ or $1.5 \mathrm{~h}$, over a time interval of $\sim 12.5 \mathrm{~h}$, which sample the rotational light curve. The sampling scheme insured that i) the light curve was adequately sampled and ii) at least two extrema were well determined, independently of the starting time. The geometric parameters varied only slightly during this interval (Table 1). The heliocentric distance changed from 4.476 to $4.478 \mathrm{AU}$, the SST-comet distance from 4.044 to $4.038 \mathrm{AU}$, and the solar phase angle from 12.12 to $12.07^{\circ}$. The image scale, based on an average pixel scale of 2.55 arcsec pixel $^{-1}$ of the MIPS $24 \mu \mathrm{m}$ channel, decreased from 7478.5 to $7467.8 \mathrm{~km} \mathrm{pixel}^{-1}$. During each visit, we acquired three cycles of fourteen dithered images with an individual exposure time of $2.62 \mathrm{~s}$. The comet nucleus was centered in the dither pattern and the telescope tracked the comet with its computed non-sidereal rates. The cumulated exposure time corresponding to the 42 images equals $110 \mathrm{~s}$. Figure 2 displays the sum of all 16 images recentered properly on the nucleus. A bright central core of emission is indeed clearly seen in the image superimposed on a narrow linear structure. This extended dust emission was analyzed in MIPS images also acquired in Feb. 2004 by Kelley et al. (2008). The emission was found to be composed of old tail grains $(\approx 590$ days old $)$ and the comet's dust trail (>1750 days old). The distinction between tail and trail in our analysis is not important and we simply refer to the dust "trail" since the emission is narrow and concentrated along the projected orbit of the comet. The important point in our analysis below is that both components are old, which implies that they do not vary on a timescale of a few hours. 
Table 1. Journal of observations of 67P/Churyumov-Gerasimenko.

\begin{tabular}{ccccccccc}
\hline \hline$\#$ & $\begin{array}{c}\text { UT date } \\
\text { 2004 February }\end{array}$ & $\begin{array}{c}r_{\mathrm{h}} \\
(\mathrm{AU})\end{array}$ & $\begin{array}{c}\Delta \\
(\mathrm{AU})\end{array}$ & $\begin{array}{c}\alpha \\
\left({ }^{\circ}\right)\end{array}$ & $\begin{array}{c}\text { Pixel } \\
(\mathrm{km})\end{array}$ & $\begin{array}{c}\text { Flux } \\
(\mathrm{mJy})\end{array}$ & $\begin{array}{c}\sigma_{1} \\
(\mathrm{mJy})\end{array}$ & $\begin{array}{c}\sigma_{2} \\
(\mathrm{mJy})\end{array}$ \\
\hline 1 & 25.0123 & 4.476 & 4.044 & 12.12 & 7478.5 & 3.36 & 0.04 & 0.08 \\
2 & 25.0196 & 4.476 & 4.044 & 12.12 & 7478.3 & 3.31 & 0.07 & 0.09 \\
3 & 25.0376 & 4.476 & 4.043 & 12.11 & 7477.9 & 3.26 & 0.16 & 0.18 \\
4 & 25.0788 & 4.476 & 4.043 & 12.11 & 7477.1 & 3.31 & 0.05 & 0.08 \\
5 & 25.1157 & 4.476 & 4.042 & 12.11 & 7476.3 & 3.26 & 0.05 & 0.08 \\
6 & 25.1347 & 4.476 & 4.042 & 12.11 & 7475.9 & 3.26 & 0.13 & 0.15 \\
7 & 25.1522 & 4.476 & 4.042 & 12.10 & 7475.6 & 3.56 & 0.04 & 0.08 \\
8 & 25.1845 & 4.476 & 4.042 & 12.10 & 7474.9 & 4.07 & 0.06 & 0.10 \\
9 & 25.2418 & 4.477 & 4.041 & 12.10 & 7473.7 & 4.28 & 0.26 & 0.27 \\
10 & 25.2796 & 4.477 & 4.041 & 12.09 & 7472.9 & 4.33 & 0.17 & 0.19 \\
11 & 25.2972 & 4.477 & 4.040 & 12.09 & 7472.6 & 3.56 & 0.11 & 0.13 \\
12 & 25.3146 & 4.477 & 4.040 & 12.09 & 7472.2 & 3.67 & 0.15 & 0.16 \\
13 & 25.3520 & 4.477 & 4.040 & 12.09 & 7471.5 & 3.06 & 0.09 & 0.11 \\
14 & 25.4753 & 4.478 & 4.038 & 12.07 & 7468.9 & 3.77 & 0.19 & 0.20 \\
15 & 25.4826 & 4.478 & 4.038 & 12.07 & 7468.8 & 3.62 & 0.09 & 0.12 \\
16 & 25.5300 & 4.478 & 4.038 & 12.07 & 7467.8 & 3.36 & 0.05 & 0.09 \\
\hline
\end{tabular}

Note:

\#: sequential number of observations.

$r_{\mathrm{h}}, \Delta$ : heliocentric, SST-centric distances of the comet.

$\alpha$ : solar phase angle.

$\sigma_{1}: 1 \sigma$ error from background and fitting.

$\sigma_{2}:$ total $1 \sigma$ error.

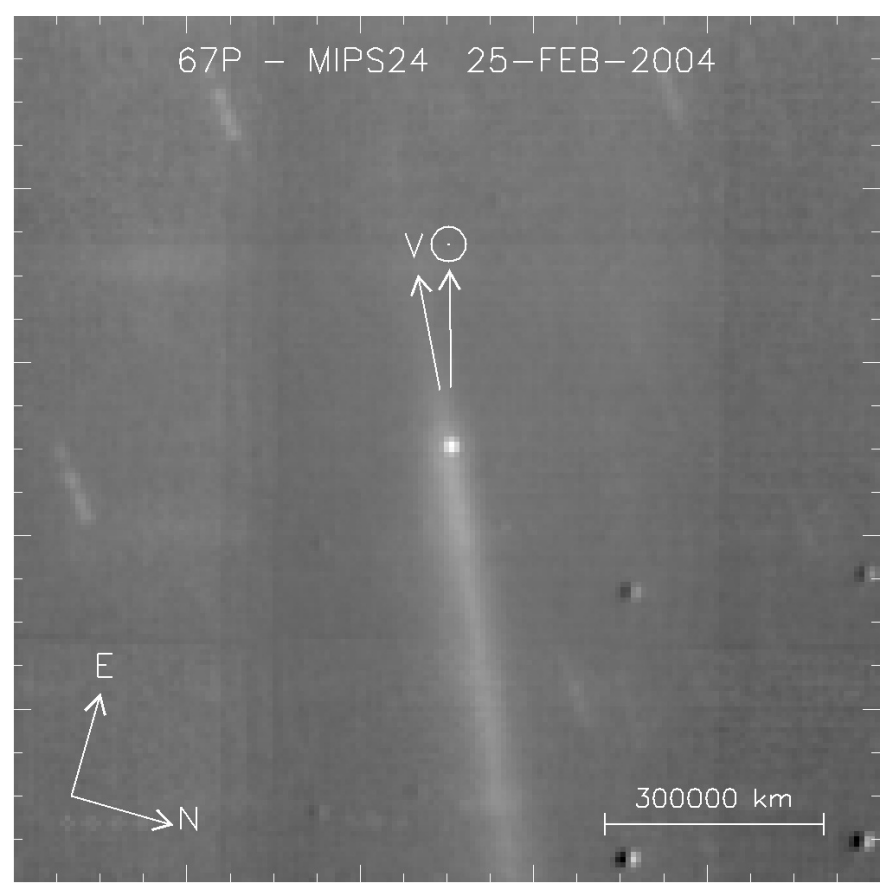

Fig. 2. Image of comet 67P/Churyumov-Gerasimenko obtained with the MIPS $24 \mu \mathrm{m}$ channel of the Spitzer space telescope on 25 February 2004. The arrows indicate the anti-solar direction (prolonged radius vector $r$ ), the heliocentric orbital velocity vector $\left(V_{\text {orb }}\right)$ of the comet projected onto the sky plane, the direction of celestial North $(\mathrm{N})$ and East (E).

In our first analysis (Lamy et al. 2004b; see also Lamy et al. 2007), the MIPS images were processed with the Spitzer Science Center's pipeline version $S 11.4$.0 producing individual basic calibrated data (BCD) frames. These BCD images were subsequently mosaicked by the MIPS team at Steward Observatory (Stansberry and Sue). In the present analysis, the raw data frames were reprocessed with the latest version of the MIPS pipeline (S14.4.0) to produce a new set of basic calibrated data. For each comet visit, we then divided each image by the median of all 42 images acquired during that visit, as prescribed by the Spitzer Science Center to remove both small scale detector artifacts and large scale gradients in the flat-field. We ensured that the background of each image agreed with all others by adding constant offsets to each image; we then mosaicked the images in the rest frame of the comet with the MOPEX software (Makovoz et al. 2005), using a final pixel scale of 2.55 arcsec pixel ${ }^{-1}$. Bad pixels, that is those permanently damaged, or affected by cosmic rays, were ignored during the mosaicking step.

\section{Data analysis}

\subsection{Background determination}

The local background levels below the nucleus and the trail are not uniform, and were estimated by introducing two thin rectangles adjacent to the trail on both sides, which had long sides orientated along the direction of the trail. Averaging the pixel values along the short sides enabled two mean profiles to be constructed, which characterized the background on both sides of the trail. We experimented by fitting different types of twodimensional surfaces to these profiles, concluding that a simple linear interpolation was adequate. This procedure was applied independently to each image, and the corresponding background level was subtracted. Figure 3 displays profiles centered on the peak pixel and extracted along and across the trail of the 16 images. The residual variations in the background levels between images are within the noise. We note in addition that the trail brightness remains constant during the sequence of observations, which is expected for an old, evolved, dust structure. In contrast, conspicuous variations in the peak pixel intensity are observed, which can be attributed to the varying cross-section of the nucleus.

\subsection{Model fitting}

As we have already noted, the nucleus appears as a bright point source superimposed on the dust trail; the point-spread function 

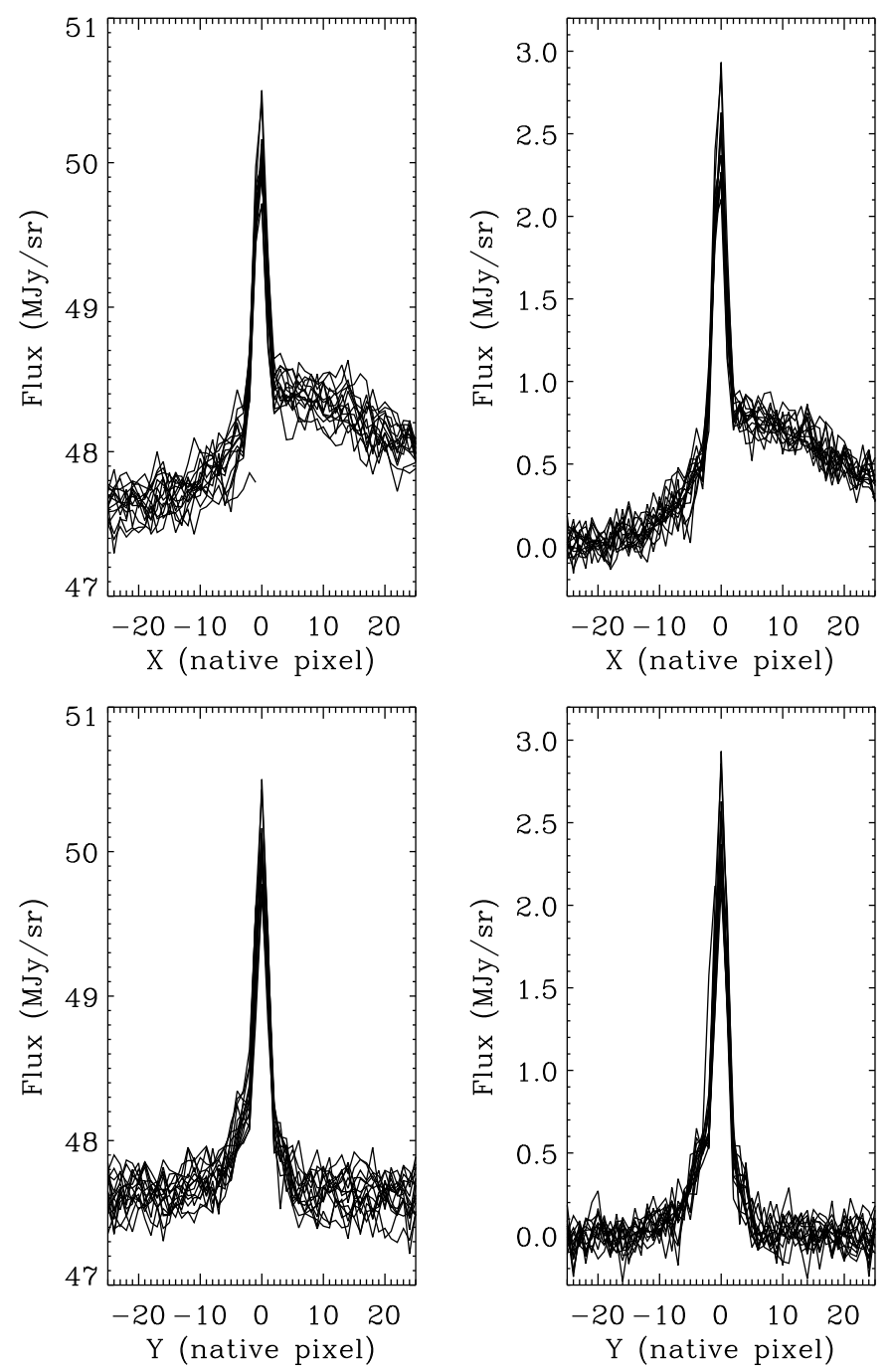

Fig. 3. Stacked $x$ and $y$-profiles of all 16 images across the nucleus. The left column corresponds to the original images while the right column to the images corrected for the non-uniform background.

fitting technique must therefore be applied to ensure that the signal of the nucleus is recovered correctly. The two-dimensional surface brightness model to be fitted to the observed images is given by:

Model $=$ Nucleus $\otimes \mathrm{PSF}+$ Trail

where $P S F$ represents the point spread function of the telescope and $\otimes$ represents the convolution operator. The nucleus is unresolved so that

nucleus $=k_{\mathrm{n}} \delta(\rho)$

where $\delta$ is the Dirac delta function, $\rho$ the projected radial distance from the center, and $k_{\mathrm{n}}$ a scaling factor. Unlike cometary comae, which can generally be described by simple physical isotropic models of the form

$\operatorname{coma}=k_{\mathrm{c}} \rho^{p}$

(Lamy et al. 2004a) or even anisotropic models by allowing the scaling factor $k_{\mathrm{c}}$ (and possibly the exponent $p$ ) to be a function(s) of the azimuth angle (see Lamy et al. 1998b, for the case of comet 19P/Borrelly), we do not have a general canonical model of cometary dust trails based on simple physical considerations.
Cometary trails must therefore be modeled from the observations themselves and consequently the term "Trail" is left outside the convolution operation in Eq. (1).

In our first analysis (Lamy et al. 2004b), the bright star HD159330, a $24 \mu \mathrm{m}$ calibrator star, was used as a PSF. The data were acquired during the same campaign as and within a few days of the observations of $67 \mathrm{P} / \mathrm{C}-\mathrm{G}$. The final image with a subsampling factor of 4 was prepared by the MIPS group at Steward Observatory. We removed a small background level by monitoring the growth curve (the encircled energy within a circular aperture versus its radius), and recording the asymptotic behavior.

Since its initial consideration, our understanding of the MIPS PSF has developed significantly. A modeling tool is available (Krist 2006), which was calibrated by extensive comparison with observations (Engelbracht et al. 2007). For the present analysis, we rely on a modeled PSF as we did before when analyzing Hubble Space Telescope observations of cometary nuclei. We used version 2.0 of the STinyTIM software written by Krist (2006) and followed the prescriptions of Engelbracht et al. (2007). STinyTIM enables PSFs to be generated taking into account the color of the observed object $(180 \mathrm{~K}$ for the nucleus of $67 \mathrm{P} / \mathrm{C}-\mathrm{G})$, using a default pixel scale of $2.55 \mathrm{arcsec}^{\mathrm{p} i x e l^{-1}}$ with subsampling factor from 1 to 10 . However, the model PSFs are significantly sharper than the images delivered by MIPS, and must be smoothed by a factor of 1.8 to reproduce the observed PSFs well, which is attributed almost entirely to the pixel sampling. To complete this operation practically, we generated STinyTIM PSFs that were oversampled by a factor of 10 and then smoothed by a boxcar of 18 pixels wide. Although we had to work with resampled PSFs to account for the the sub-pixel location of the nucleus (which can cause a marked asymmetry of the brightness profiles), a factor as large as 10 is not warranted for this purpose and we rebinned them by a factor 2.5 (we used the IDL CONGRID function with the linear interpolation). The final PSF used in our analysis has an oversampling factor of 4 relative to the pixel scale of $2.55 \mathrm{arcsec}_{\mathrm{pixel}}^{-1}$, which implies that there are 16 possibilities for the sub-pixel location of the nucleus. The fit to the observed images was performed after integrating the model over $4 \times 4$ sub-pixels to recover the original "native" MIPS pixel. On each image, the sub-pixel coordinates of the nucleus $\left(x_{\mathrm{n}}, y_{\mathrm{n}}\right)$ were determined using the $x$ and $y$-profiles across the pixels displaying the maximum signal.

We adopted an empirical approach in constructing a model of the trail: this consisted of subtracting properly centered PSFs from the observed images and tuning progressively the model parameter $k_{\mathrm{n}}$ so as to produce a regular structure without any strong gradients, consistent with our expectation for an evolved dust feature. We average-combined the sixteen images, which removed successfully small imperfections present in the individual fits. By applying this robust model of the trail, we performed a final fit of our model given by Eq. (1) to the observed images to determine the optimal values of the parameter $k_{\mathrm{n}}$. Figure 4 provides two examples of the fit quality corresponding to extreme cases where the nucleus is either badly off-centered or well centered in a pixel. The optimization was performed by minimizing the residuals both along the $x$ and $y$-profiles, and in a circular aperture of diameter 5 pixels (see the histograms of the residuals in the middle panels of the figures).

The absolute calibration was performed using a calibration factor of $4.47 \times 10^{-2} \mathrm{MJy}(\mathrm{DN} / \mathrm{s})^{-1} \mathrm{sr}^{-1}$. Since the PSF used in our analysis is normalized such that its total integral is equal to 1 , an aperture correction is not required and the flux of the nucleus is derived by multiplying the $k_{\mathrm{n}}$ parameters with a 

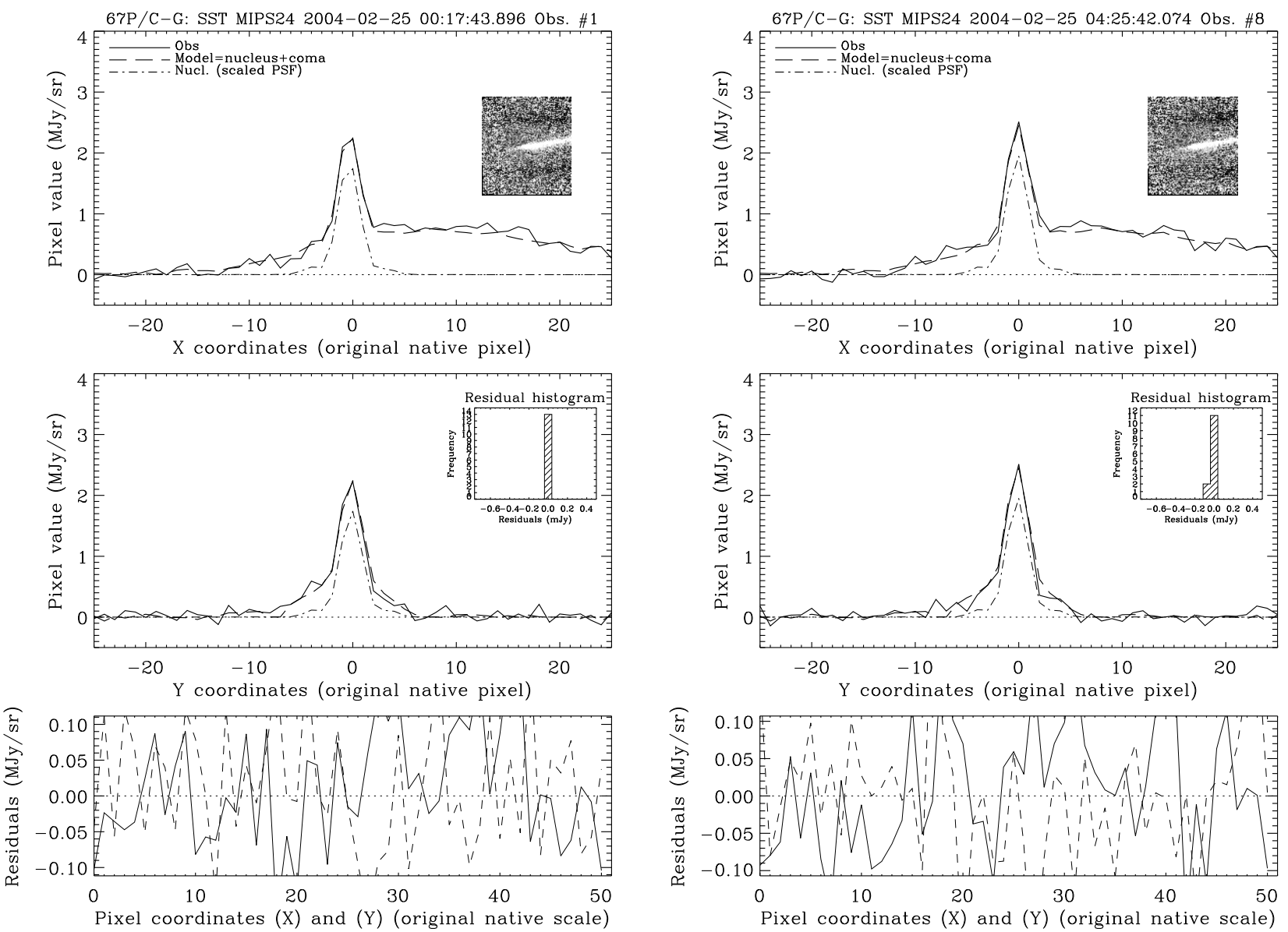

Fig. 4. Left: fit of the model to the first image. The top panel displays the $x$-profiles and the middle panel the $y$-profiles, which are both across the nucleus. The bottom panel displays both profiles of the residuals. The histogram of the residuals limited to the central core of the PSF is inserted in the middle panel. Right: similar data to the left panel for the 8th observation.

coefficient determined by both the above calibration factor and the spatial extent of the pixel in steradians. A final correction is required since the calibration factor is valid for a temperature of $10^{4} \mathrm{~K}$. The fluxes were divided by 0.938 that we interpolated from Table 8.3 of the Spitzer Observer's Manual (Version 8.0, $2007)$ for the temperature ( $180 \mathrm{~K})$ of the nucleus.

The principal errors affecting the corresponding fluxes originate in the determination of the background and in the fitting process. We estimated the $1 \sigma$ uncertainty in the background level to be $0.015 \mathrm{mJy}$ from its statistical fluctuations. The error generated by the fitting procedure is measured by assessing the residuals as described above; it is expressed as a fraction of the scaling factors and ranges typically between 2 and $6 \%$. As recommended by Engelbracht et al. (2007), we adopted an uncertainty in the calibration factor of $2 \%$. It is unlikely that the performance of MIPS varied significantly during $12 \mathrm{~h}$ and this error can be assumed to be systematic; it is irrelevant when considering the relative variation in the light curve, but is introduced in the determination of the physical parameters of the nucleus.

Table 1 summarizes our results with two different $(1 \sigma)$ uncertainties affecting the fluxes of the nucleus. The first uncertainty includes only the cumulated error due to uncertainties in both the background determination and the fitting process; it is therefore relevant to the relative variation in the light curve as displayed in Fig. 5. The second uncertainty includes in addition the error in the calibration factor.
The shape of this new thermal light curve is similar to that derived from our first analysis (Lamy et al. 2004b, 2007): a couple of data points are shifted without affecting the global behavior of the temporal variation. It is puzzling that we do not observe a well-behaved, double-peaked light curve consistent with those found in the visible (Lamy et al. 2006), and this will become more obvious when a comparison is completed with thermal models. Even if the extraction of the nucleus is imperfect, the temporal variation in its flux should be preserved because the underlying dust trail is stable. We note that an SST observation with MIPS $(24 \mu \mathrm{m})$ and IRAC $(8 \mu \mathrm{m})$ performed by D. Wooden and coworkers (SST DDT 294) in May 2007 under similar geometric conditions as in February 2004 produce even more puzzling light curves with a single extremum over a time interval of $14 \mathrm{~h}$ (Kelley, private communication). The thermal light curve is a combination of shape effects and thermal properties; since the shape is prominently controlled by the visible observations, the thermal light curve would require highly non-uniform properties of the nucleus. We presently have no explanation for this situation which calls for additional observations.

A final comment concerns the increase in flux by approximately $30 \%$ between our preliminary and present data reductions. This is understood to be caused by the different PSFs $(\sim 15 \%)$ and a difference between pixel sizes used for resampling the cometary and star images. 


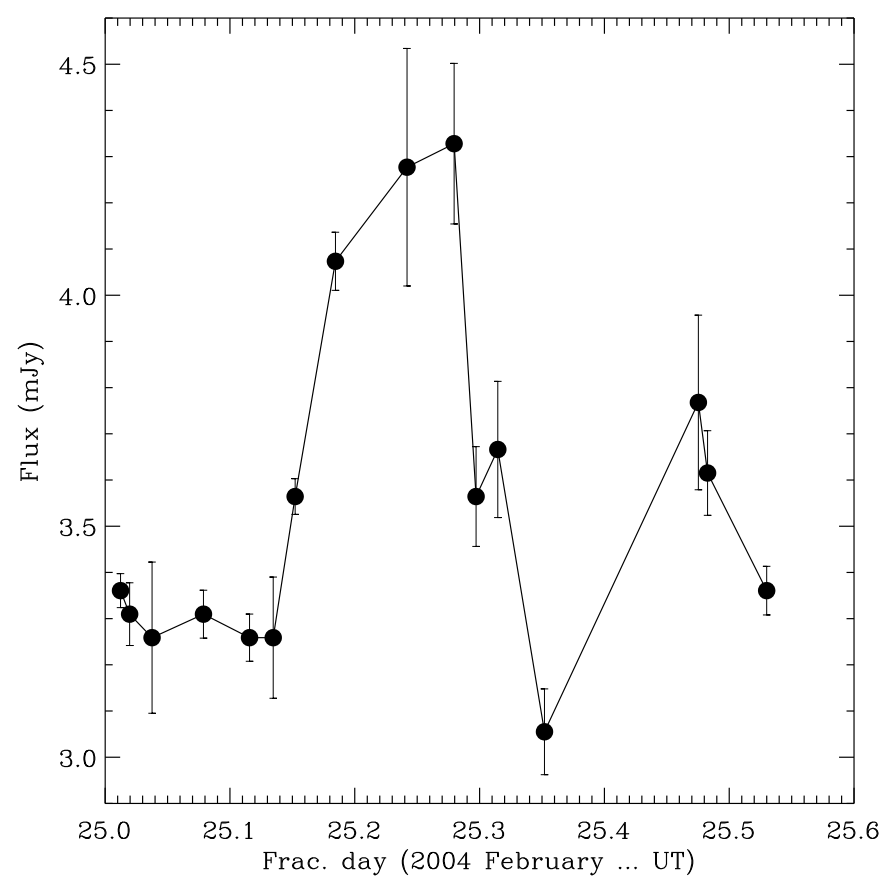

Fig. 5. The thermal light curve of the nucleus of comet $67 \mathrm{P} / \mathrm{Churyumov}$ Gerasimenko at $24 \mu \mathrm{m}$. The error bars correspond to $\sigma_{1}$ as listed in Table 1.

Table 2. Results for the synodic period $P_{\text {rot }}$.

\begin{tabular}{lcc}
\hline \hline Method & \multicolumn{2}{c}{$P_{\text {rot }}$} \\
\cline { 2 - 3 } & (day) & (hour) \\
\hline Autocorrel. MAC & 0.582 & 14.0 \\
Lomb-Scargle & 0.662 & 15.9 \\
DFT & 0.763 & 18.3 \\
WindowCLEAN & 0.788 & 18.9 \\
PDM & 0.806 & 19.3 \\
AoV & 0.926 & 22.2 \\
Inform. entropy & 0.978 & 23.5 \\
\hline
\end{tabular}

\section{Analysis and interpretation}

\subsection{Period analysis}

The thermal light curve of the nucleus of $67 \mathrm{P} / \mathrm{C}-\mathrm{G}$ was analyzed using seven different period-searching methods, which were introduced in our previous analysis of HST data (Lamy et al. 2006):

1. the modified autocorrelation (MAC) technique described by Burki et al. (1978);

2. the information entropy spectrum of a time series, as defined by Cincotta et al. (1995);

3. the power spectrum of the data using Kurtz's (1985) implementation of Deeming's (1975) discrete Fourier transform (DFT) algorithm for unequally-spaced data;

4. the Lomb-Scargle method (Lomb 1976; Scargle 1982);

5. the period dispersion minimization method (PDM, Stellingwerf 1978);

6. the analysis of variance (AoV) method (SchwarzenbergCzerny 1989, 1991), which improves and corrects the original version of the PDM method (Stellingwerf 1978);

7. the WindowCLEAN method (Belton \& Gandhi 1988).

The original flux data were used as an input to the period analysis without any correction for the (negligible) changes in the

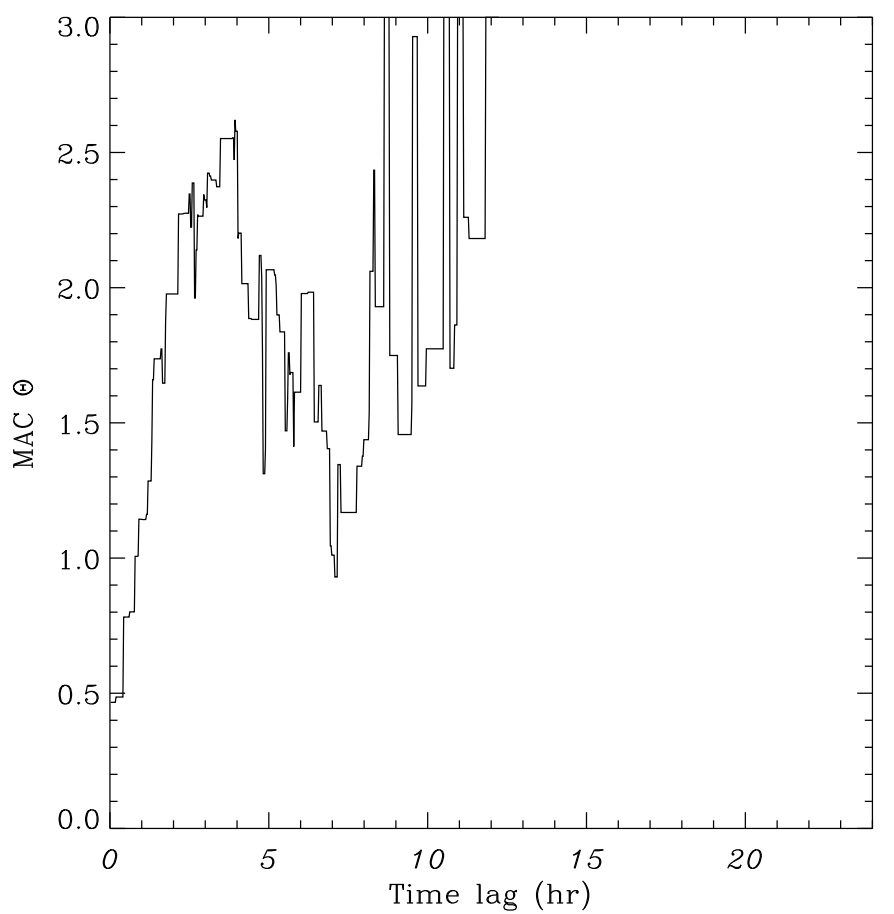

Fig. 6. Autocorrelation periodogram of the thermal-infrared light curve for the nucleus of 67P/Churyumov-Gerasimenko using the modified autocorrelation method (MAC $\Theta$ ). The most probable sine period is at the first minimum of the periodogram.

heliocentric and SST-centric distances during the observational interval; they were simply shifted to a mean level of zero. The results are presented in Table 2, and the large scatter between 14 and $23.5 \mathrm{~h}$ is a direct consequence of the odd shape of the thermal light curve, which departs strongly from the expected double-peaked variation as discussed already in the above section. We note that the autocorrelation analysis, which is known to be suitable for detecting quasi-periodic trends in a time series and to yield generally robust results, produces i) a well-defined minimum in the periodogram (Fig. 6); and ii) a synodic rotational period of $14 \mathrm{~h}$, which is the closest in value to the range of 12.4-12.7 h that we derived from visible observations (Lamy et al. 2006).

\subsection{Thermal model}

We interpret our infrared using our thermal model developed in many previous works (Lamy et al. 2002; Groussin \& Lamy 2003; Groussin et al. 2004), which we now describe briefly. The surface of the nucleus is divided into $\sim 1000$ facets, and for each one, we solve for the surface energy balance between the flux received from the Sun, the re-radiated flux, and the heat conduction into the nucleus. As the nucleus rotates around its spin axis, the illumination changes and the heat conduction equation is solved at each facet for a one-dimensional, time-dependent temperature. The time step ( $\sim 170 \mathrm{~s})$ is sufficiently small to ensure numerically accurate solutions. Given the considered nucleus parameters, the model typically relaxes in 30 rotations, i.e., $T(t)$ no longer changes from one cycle to the next. As a result, we were able to obtain the temperature of each facet as a function of time, over one period of rotation. From this temperature profile, we calculated the infrared flux $F_{\mathrm{i}}$ from each facet as a function of time. The total flux received by the observer is then the sum of the individual fluxes $F_{\mathrm{i}}$ of all facets of the shape model. We 
considered two models for the shape of the nucleus, namely solutions $\mathrm{A}$ and $\mathrm{B}$, yielded by the simultaneous inversion of a set of visible light curves (Lamy et al. 2007).

\subsection{Parameters of the thermal model}

Our model has six free parameters: the infrared emissivity $\epsilon$, the phase integral $q$, the scaling coefficient $\gamma$, the geometric albedo $p_{V}$, the beaming factor $\eta$, and the thermal inertia $I$. Among these six parameters, we consider that three of them $\epsilon, q$, and $p_{V}$ could be reasonably assumed while the other three $\gamma, \eta$, and $I$ must be assumed or constrained from the observational results.

The infrared emissivity $\epsilon$ is assumed to be 0.95 , which is the middle point of the interval $0.9-1.0$ generally quoted in the literature. Since the interval is small and close in value to 1.0, this uncertainty has a negligible influence on the calculated thermal flux.

The phase integral $q$ measures the angular dependence of the scattered radiation. We chose $q=0.27$, the value measured for 19P/Borrelly by Buratti et al. (2004).

The parameter $\gamma$ scales the model flux to ensure that it is able to reproduce the data, and controls the size of the nucleus directly. As a consequence, it can be determined independently for each combination of $\eta$ and $I$.

The beaming factor $\eta$ was defined by Lagerros (1998) and reflects only the influence of surface roughness, which produces an anisotropic thermal emission. Theoretically, $\eta$ ranges from 0 to 1 , but in practice it must be larger than 0.7 to avoid unrealistic roughness, leading to slopes with rms values exceeding 1 (Lagerros 1998). For low albedo objects such as cometary nuclei, Lagerros (1998) found that a rather high surface roughness is required to achieve the standard value of 0.756 from Lebofsky et al. (1986): following his recommendation, we selected the more appropriate value of $\eta=0.85$.

Finally, we considered three values of the thermal inertia $I=$ $0,10,50 \mathrm{~J} \mathrm{~K}^{-1} \mathrm{~m}^{-2} \mathrm{~s}^{-1 / 2}$, which cover a range consistent with the result found for 9P/Tempel 1 (Groussin et al. 2007).

\subsection{Size of the nucleus}

Using our solutions $\mathrm{A}$ and $\mathrm{B}$ for the three-dimensional shape of the nucleus of $67 \mathrm{P} / \mathrm{C}-\mathrm{G}$ and its rotational state derived from visible observations (Lamy et al. 2007), we calculated the corresponding thermal light curves at $24 \mu \mathrm{m}$ for the different values of the thermal inertia, taking into account the geometric conditions of our SST observations. As expected, the double-peaked synthetic light curves cannot be fitted to the entire set of data points, and we adjusted the phase shift and the scaling factor $\gamma$ on the observed broad maximum, achieving residuals of $\sim 3 \%$ (Fig. 7). The scaling factor $\gamma$ was determined to an accuracy of $2 \%$. For the data points that are outliers of the best-fit relations for the light curves, the residuals do not exceed $\sim 15 \%$ for solution $\mathrm{A}$ and $\sim 25 \%$ for solution $\mathrm{B}$. Therefore, the apparent discrepancy, although puzzling, does not have a dramatic impact on the quantitative determination of the physical parameters; the results can even be considered satisfactory in view of the complexity of the overall procedure. The determination of the parameter $\gamma$ then enables our three-dimensional models of the shape of the nucleus to be scaled, and the results are reported in Table 3. As long as the thermal inertia does not exceed $\sim 20 \mathrm{~J} \mathrm{~K}^{-1} \mathrm{~m}^{-2} \mathrm{~s}^{-1 / 2}$, the size of the nucleus is well constrained, and the overall dimensions measured along the principal axis are 5.1-5.3 km, 4.2-4.3 km, and 3.4-3.6 km for solution A, and

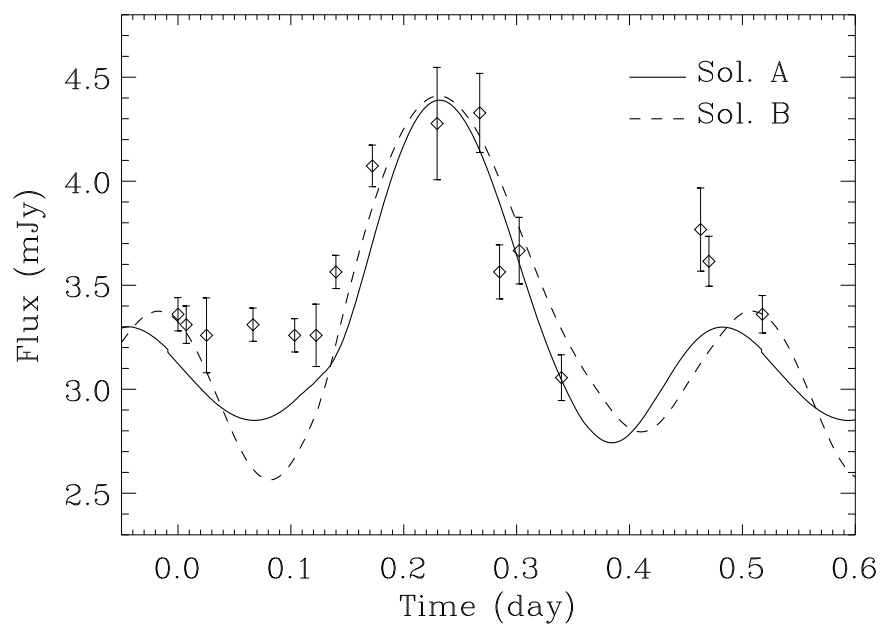

Fig. 7. The calculated thermal infrared light curves of the nucleus of comet 67P/Churyumov-Gerasimenko at $24 \mu \mathrm{m}$ for the two shape models to be compared with the MIPS data. The error bars correspond to $\sigma_{2}$ as listed in Table 1.

Table 3. Size of the nucleus of 67P.

\begin{tabular}{cccccc}
\hline \hline$I$ & $\begin{array}{c}\text { Shape } \\
\text { model }\end{array}$ & $\begin{array}{c}a \times b \times c \\
(\mathrm{~km})\end{array}$ & $\begin{array}{c}\text { Surface } \\
\left(\mathrm{km}^{2}\right)\end{array}$ & $\begin{array}{c}\text { Volume } \\
\left(\mathrm{km}^{3}\right)\end{array}$ & $\begin{array}{c}r_{v} \\
(\mathrm{~km})\end{array}$ \\
\hline 0 & A & $2.55 \times 2.09 \times 1.72$ & 51.4 & 30.0 & 1.93 \\
0 & B & $2.20 \times 2.08 \times 1.69$ & 52.4 & 31.0 & 1.95 \\
10 & A & $2.60 \times 2.13 \times 1.75$ & 53.5 & 31.8 & 1.97 \\
10 & B & $2.28 \times 2.16 \times 1.76$ & 56.6 & 34.9 & 2.03 \\
20 & A & $2.65 \times 2.17 \times 1.79$ & 55.7 & 33.8 & 2.01 \\
20 & B & $2.35 \times 2.22 \times 1.81$ & 59.9 & 37.9 & 2.08 \\
30 & A & $2.73 \times 2.23 \times 1.84$ & 58.9 & 36.7 & 2.06 \\
30 & B & $2.43 \times 2.30 \times 1.87$ & 64.1 & 42.1 & 2.16 \\
40 & A & $2.80 \times 2.29 \times 1.89$ & 62.1 & 39.8 & 2.12 \\
40 & B & $2.49 \times 2.36 \times 1.92$ & 67.3 & 45.2 & 2.21 \\
50 & A & $2.87 \times 2.35 \times 1.91$ & 65.3 & 42.9 & 2.17 \\
50 & B & $2.57 \times 2.43 \times 1.98$ & 71.6 & 49.6 & 2.30 \\
\hline
\end{tabular}

Thermal inertia $(I)$ is in $\mathrm{J} \mathrm{K}^{-1} \mathrm{~m}^{-2} \mathrm{~s}^{-1 / 2}$.

4.4-4.7 km, 4.2-4.4 km, and 3.4-3.6 km for solution B. These are our favored solutions because we expect the nucleus of comet $67 \mathrm{P} / \mathrm{C}-\mathrm{G}$ to have a low thermal inertia, especially if a bulk density in the range $0.1-0.37 \mathrm{~g} \mathrm{~cm}^{-3}$ (Davidsson \& Gutiérrez 2005) is confirmed. The corresponding surface area equals $51-60 \mathrm{~km}^{2}$ and the volume $30-38 \mathrm{~km}^{3}$, which correspond to an effective radius (that of the sphere of identical volume) of $1.93-2.08 \mathrm{~km}$. "Extreme" solutions with a thermal inertia of $50 \mathrm{~J} \mathrm{~K}^{-1} \mathrm{~m}^{-2} \mathrm{~s}^{-1 / 2}$ have an effective radius of $r_{\mathrm{n}, v}=2.17-2.30 \mathrm{~km}$, which is larger than our nominal solutions by only $10-13 \%$.

Almost simultaneously to our observations on 23 February 2004, Kelley et al. (2008) used the same instrumental configuration (MIPS at 24 micron) to perform a snapshot observation of $67 \mathrm{P} / \mathrm{C}-\mathrm{G}$. The nucleus flux was derived by fitting a PSF derived from nearby stars to the comet's central point source, and the application of a near-Earth asteroid thermal model (NEATM) yielded a radius of $1.87 \pm 0.08 \mathrm{~km}$. As pointed out by Lamy et al. (2004a), a snapshot observation provides, on average, a good estimate of the effective radius of the equivalent sphere. The agreement with our present nominal solution is therefore excellent. 


\subsubsection{Albedo}

Originally devised by Russell (1916) for asteroids and conveniently reformulated by Jewitt (1991), the standard formula relating magnitudes to cross-sections was applied in calculating the geometric albedo $p$ in a given photometric band. This formula also incorporates the phase function. We relied on our HST observations, which were acquired at a solar phase angle of $4.8^{\circ}$; this is consistent with our discussion in Lamy et al. (2007) and excludes the opposition surge that translates into an artificial increase of the albedo. Based on the similarity between the phase functions of $67 \mathrm{P} / \mathrm{C}-\mathrm{G}$ and $9 \mathrm{P} /$ Tempel 1 , we adopted a phase coefficient $\beta=0.0465 \mathrm{mag} / \mathrm{deg}$ for the linear part of the phase function. The resulting peak magnitude $R(1,1,0)=15.46$ then corresponds to the maximum geometric cross-section observed by HST at the time of the observations and is calculated for our two 3-D models of the nucleus, taking into account the proper geometric conditions. The results are displayed in Fig. 8. As long as the thermal inertia does not exceed $\sim 20 \mathrm{~J} \mathrm{~K}^{-1} \mathrm{~m}^{-2} \mathrm{~s}^{-1 / 2}$, the albedo remains well constrained in the range 0.040-0.043 for solution A and 0.037-0.042 for solution B, remarkably consistent with the canonical value of 0.04 for cometary nuclei. A thermal inertia of $50 \mathrm{~J} \mathrm{~K}^{-1} \mathrm{~m}^{-2} \mathrm{~s}^{-1 / 2}$ decreases the albedo to $\sim 0.03$, which is still in the accepted range of albedos (Fernandez et al. 2001; Lamy et al. 2004a). We note that the HST magnitudes measured at a phase angle $\alpha=4.8^{\circ}$ could be biased by the opposition effect and that a putative correction of 0.1 mag would decrease the above range to $0.034-0.039$. To highlight the effect of the strong opposition effect on the albedo, we finally repeated the above calculation but for a $\mathrm{H}-\mathrm{G}$ phase function with $G=-0.45$ as determined by Lamy et al. (2007). The albedo values were naturally larger, by a factor 1.65 (Fig. 8).

\section{Conclusions}

We have presented a refined analysis of our Spitzer Space Telescope observations of comet $67 \mathrm{P} /$ Churyumov-Gerasimenko, which is the target of the Rosetta mission. The observations were performed in February 2004 and analyzed on the basis of an improved understanding of the performances of the MIPS instrument. The main results of our revised analysis is an upward revision of the thermal flux of the cometary nucleus, leading to a slightly larger size than for our preliminary analysis (Lamy et al. 2007). Accordingly, the albedo is revised downward, well in line with the canonical value for cometary nuclei. Our main findings are summarized below.

1. The thermal light curve ranges from 3.06 to $4.3 \mathrm{mJy}$ but does not clearly exhibit the double-peaked behavior expected from our visible observations.

2. Accordingly, the synodic rotational period is poorly constrained. The autocorrelation analysis yielded $P_{\text {rot }}=14 \mathrm{~h}$, which is the value closest to the range of $12.4-12.7 \mathrm{~h}$ derived from visible observations.

3. We favor a solution with a low thermal inertia that constraints well the size of the nucleus: the overall dimensions measured along the principal axis are then $4.40-5.20 \mathrm{~km}$, $4.16-4.30 \mathrm{~km}$, and $3.40-3.50 \mathrm{~km}$. The corresponding effective radius, for a sphere of an identical volume, is in the range $1.93-2.03 \mathrm{~km}$. The "extreme" solution would have an effective radius of $2.3 \mathrm{~km}$.

4. Our nominal solution has an albedo in the range 0.039-0.043, remarkably consistent with the canonical value of 0.04 for cometary nuclei.
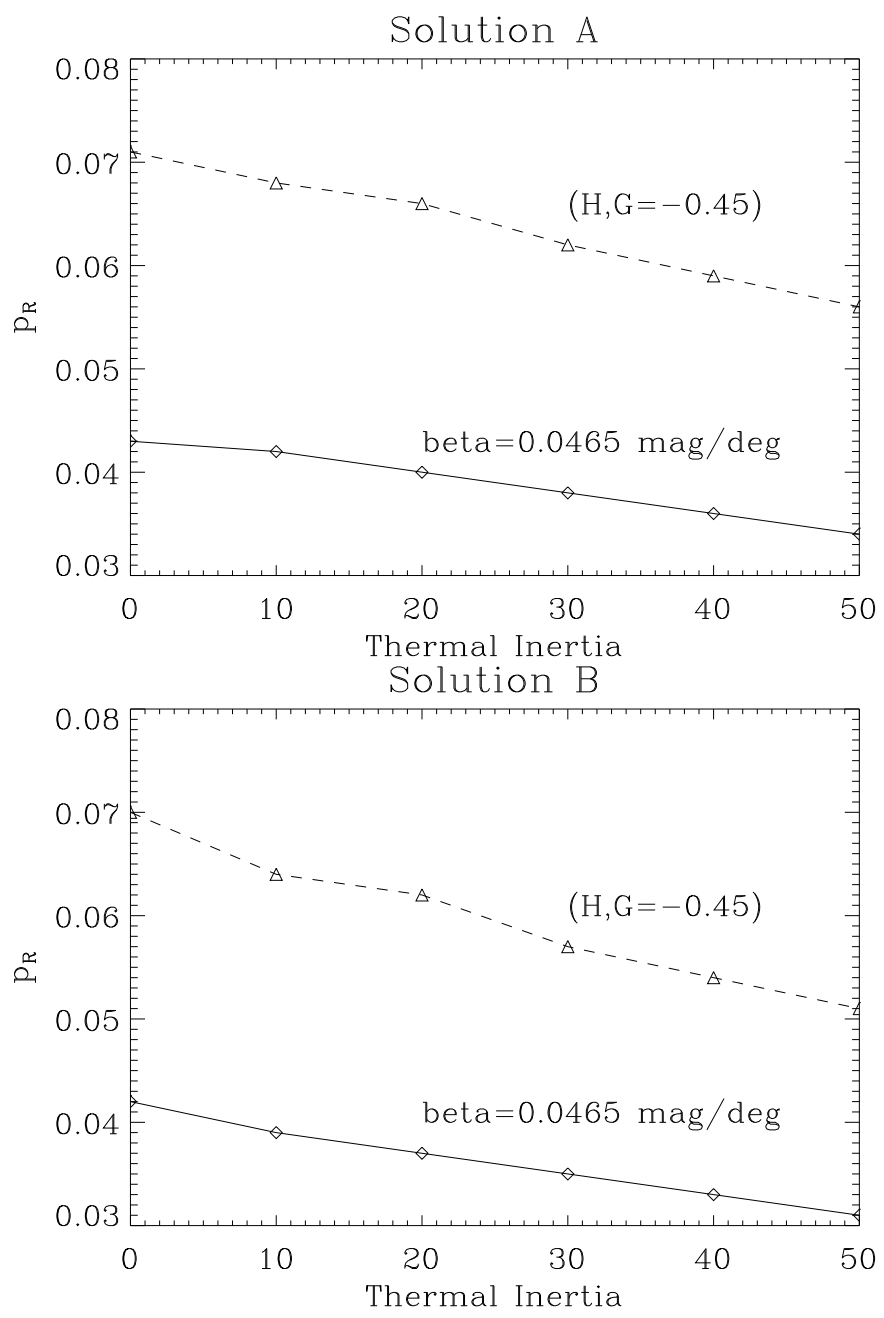

Fig. 8. $R$-band albedo of the nucleus of comet 67P/ChuryumovGerasimenko as a function of thermal inertia for the two shape models. The solid lines correspond to a linear phase law with a phase coefficient $\beta=0.0465 \mathrm{mag} / \mathrm{deg}$. The dashed lines correpond to a $\mathrm{H}-\mathrm{G}$ phase function with $G=-0.45$.

5. The success of the landing of the Philae surface module now remains critically dependent upon the bulk density of the nucleus. A value of $0.35 \mathrm{~g} \mathrm{~cm}^{-3}$, similar to that of 9P/Tempel 1 and supported by the theoretical work of Davidsson \& Gutiérrez (2005), would ensure a safe landing. A larger value, for instance $0.5 \mathrm{~g} \mathrm{~cm}^{-3}$, would place the success of the landing at risk.

Acknowledgements. We express our gratitude to T. Soifer, Director of the Spitzer Space Telescope Science Institute, for granting us Director's Discretionary time. We thank the SST ground system personnel for their prompt and efficient scheduling of the observations, and M. Hilchenbach for providing Fig. 1. I. Toth acknowledges a grant from the CNES (Centre National d'Études Spatiales) to support his stay at Laboratoire d'Astrophysique de Marseille.

\section{References}

Belton, M. J. S., \& Gandhi, A. 1988, BAAS, 20, 836, [15.06]

Buratti, B., Hicks, M. D., Soderblom, L. A., et al. 2004, Icarus, 167, 16 Burki, G., Meader, A., \& Rufener, F. 1978, A\&A, 65, 363

Carusi, A., Perozzi, E., Valsecchi, G. B., \& Kresák 1985, Long-term Evolution of Short-Period Comets (Bristol: Adam Hilger)

Cincotta, P. M., Mendez, M., \& Nunez, J. A. 1995, ApJ, 449, 231

Davidsson, B. J. R., \& Gutiérrez, P. J. 2004, Icarus, 168, 392

Davidsson, B. J. R., \& Gutiérrez, P. J. 2005, Icarus, 176, 453 
Davidsson, B. J. R., Gutiérrez, P. J., \& Rickman, H. 2007, Icarus 187, 306 Deeming, T. J. 1975, Ap\&SS, 36, 137

Ellwood, J., Schwehm, G., Rosetta Project Team, \& Bond, P. 2004, ESA Bull., 117,4

Engelbracht, C. W., Blaylock, M., Su, K. Y. L., Rho, J., et al. 2007, PASP, 119, 994

Farnham, T. L., \& Cochran, A.L. 2002, Icarus, 160, 398

Fernández, Y. R., Jewitt, D. C., \& Sheppard, S. S. 2001, ApJ, 553, L197

Groussin, O., \& Lamy, P. 2003, A\&A, 412, 879

Groussin, O., Lamy, P., Jorda, L., \& Toth, I. 2004, A\&A, 419, 375

Groussin, O., A'Hearn, M. F., Li, J.-Y., et al. 2007, Icarus, 191, 63

Harris, A. W., \& Lagerros, J. S. V. 2002, in Asteroids III, ed. W. F. Bottke, Jr., A. Cellino, P. Paolicchi, \& R. P. Binzel (Tucson: Univ. Arizona press), 205

Hilchenbach, M., Rosenbauer, H., \& Chares, B. 2004, in The New ROSETTA Targets, ed. L. Colangeli et al. (Dordrecht, Boston, London: Kluwer Acad. Pub., 289)

Jewitt, D. C. 1991, in Comets in the post-Halley era, ed. R. L. Newburn, M. Neugebauer, \& J. Rahe (Dordrecht, Boston, London: Kluwer Acad. Pub.), 19 Kelley, M. S., Reach, W. T., \& Lien, D. J. 2008, Icarus, 193, 572

Krist, J. 2006, PSF modeling for MIPS imaging: STinyTIM, the Spitzer version of the TinyTIM. http://ssc.spitzer.caltech.edu/mips/psf.html Kurtz, D. W. 1985, MNRAS, 213, 773

Lagerros, J. S. V. 1998, A\&A, 332, 1123

Lamy, P. L., Toth, I., Jorda, L., \& Weaver, H. A. 1998a, A\&A, 335, L25
Lamy, P. L., Toth, I., \& Weaver, H. A. 1998b, A\&A, 337, 945

Lamy, P. L., Toth, I., Jorda, L., et al. 2002, Icarus, 156, 442

Lamy, P. L., Toth, I., Fernández, Y. R., \& Weaver, H. A. 2004a, in Comets II, ed. M. Festou, H. U. Keller, H. A. Weaver (Tucson: Univ. Arizona press), 223

Lamy, P. L., Jorda, L., Toth, I., et al. 2004b, 35th COSPAR Scientific Assembly, 35,1824

Lamy, P. L., Toth, I., Weaver, H. A., et al. 2006, A\&A, 458, 669

Lamy, P. L., Toth, I., Davidsson, B. J. R., et al. 2007, Space Sci. Rev., 128, 23

Lebofsky, L., Sykes, M. V., Tedesco, E. F., et al. 1986, Icarus 68, 239

Lomb, N. R., 1976, ApSS, 39, 447

Makovoz, D., Khan, I., \& Masci, F. 2005, SPIE, 6065, 330

Richardson, J. E., Melosh, H. J., Lisse, C. M., \& Carcich, B. 2007, Icarus, 190, 357

Rieke, G. H., Young, E. T., Engelbracht, C. W., et al. 2004, ApJS, 154, 25

Russell, H. N. 1916, ApJ, 43, 173

Scargle, J. H. 1982, ApJ, 263, 835

Schwarzenberg-Czerny, A. 1989, MNRAS, 241, 153

Schwarzenberg-Czerny, A. 1991, MNRAS, 253, 198

Schwehm, G. H. 2003, BAAS, 35, 1001

Stellingwerf, R. F. 1978, ApJ, 224, 953

Weissman, P. R., Asphaug, E., \& Lowry, S. C. 2004, in Comets II, ed. M. C. Festou, H. U. Keller, \& H. A. Weaver (Tucson: Univ. Arizona Press, 337)

Werner, M. W., Roellig, T. L., Low, F. J., et al. 2004, ApJS, 154, 1 